\title{
Post-Traumatic Pudendal Bleeding: Beyond the Satisfaction of Search
}

\author{
Andrea Contegiacomo MD ${ }^{1}$, Anna Rita Scrofani MD², Ernesto Punzi MD² \\ and Riccardo Manfredi MD ${ }^{2}$
}

${ }^{1}$ Fondazione Policlinico Universitario A. Gemelli IRCCS, Rome, Italy

¿Università Cattolica del Sacro Cuore, Rome, Italy

Keywords: Embolization; Pelvic Trauma; Diagnostic Angiography

Received: 11 February 2021; Accepted: 28 February 2021

Vascular injury is a common (up to $40 \%$ ) finding in patients with pelvic trauma [1] and is frequently associated with multiple vessel involvement [2]. Endovascular embolization is usually the treatment of choice [3]; but, in patients with low hemoglobin levels, the vasoconstriction can obscure possible sources of bleeding by increasing procedural time or requiring re-treatments [4]. Apparent concordance between computed tomography (CT) and angiographic findings is a potential risk factor for preventing the interventional radiologist from performing a complete angiographic examination, such as selective cannulation and/or pressure injection, of the affected vascular district.

We report about a 46-year-old man with pelvic trauma following a motorcycle accident. The patient presented with signs of hemodynamic instability, low hemoglobin levels $(5.7 \mathrm{~g} / \mathrm{dl})$ and clear evidence of left pudendal artery bleeding at the CT examination performed after admission to the emergency department (Figure 1 $a-c$ ).

Right common femoral artery access was performed using a 5-French vascular sheath placement. Selective and super-selective catheterization of the left hypogastric and pudendal arteries was performed, respectively (Figure $1 d, e)$. Angiographic findings were in accordance with those reported in the previous CT, but evaluation of the contralateral pudendal artery demonstrated massive bleeding (Figure $1 f, g$ ), probably covered up by vasoconstriction, spasm or cross-circulation via anastomosis

\section{Corresponding author:}

Andrea Contegiacomo, Largo A. Gemelli 8, 00168, Rome, Italy. Email: andrea.contegiacomo@policlinicogemelli.it (C) 2021 CC BY 4.0 - in cooperation with Depts. of Cardiothoracic/ Vascular Surgery, General Surgery and Anesthesia, Örebro University Hospital and Örebro University, Sweden of the bilateral pudendal arteries, and immediately controlled with a combination of coils and sponge particle embolization (Figure $1 b-k$ ).

In conclusion, bilateral selective angiographic examination using a power injector should be performed in pelvic post-traumatic vascular injuries in order to reduce the lack of identification and treatment of vascular injuries.

\section{Ethics Statement}

(1) All the authors mentioned in the manuscript have agreed to authorship, read and approved the manuscript, and given consent for submission and subsequent publication of the manuscript.

(2) The authors declare that they have read and abided by the JEVTM statement of ethical standards including rules of informed consent and ethical committee approval as stated in the article.

\section{Conflicts of Interest}

The authors declare that they have no conflicts of interest.

\section{Funding}

The authors received no financial support for the research, authorship, and/or publication of this article.

\section{Author Contributions}

AC was responsible for writing and reviewing; ARS and EP were responsible for images and clinical data research; and RM was responsible for reviewing and supervising. 

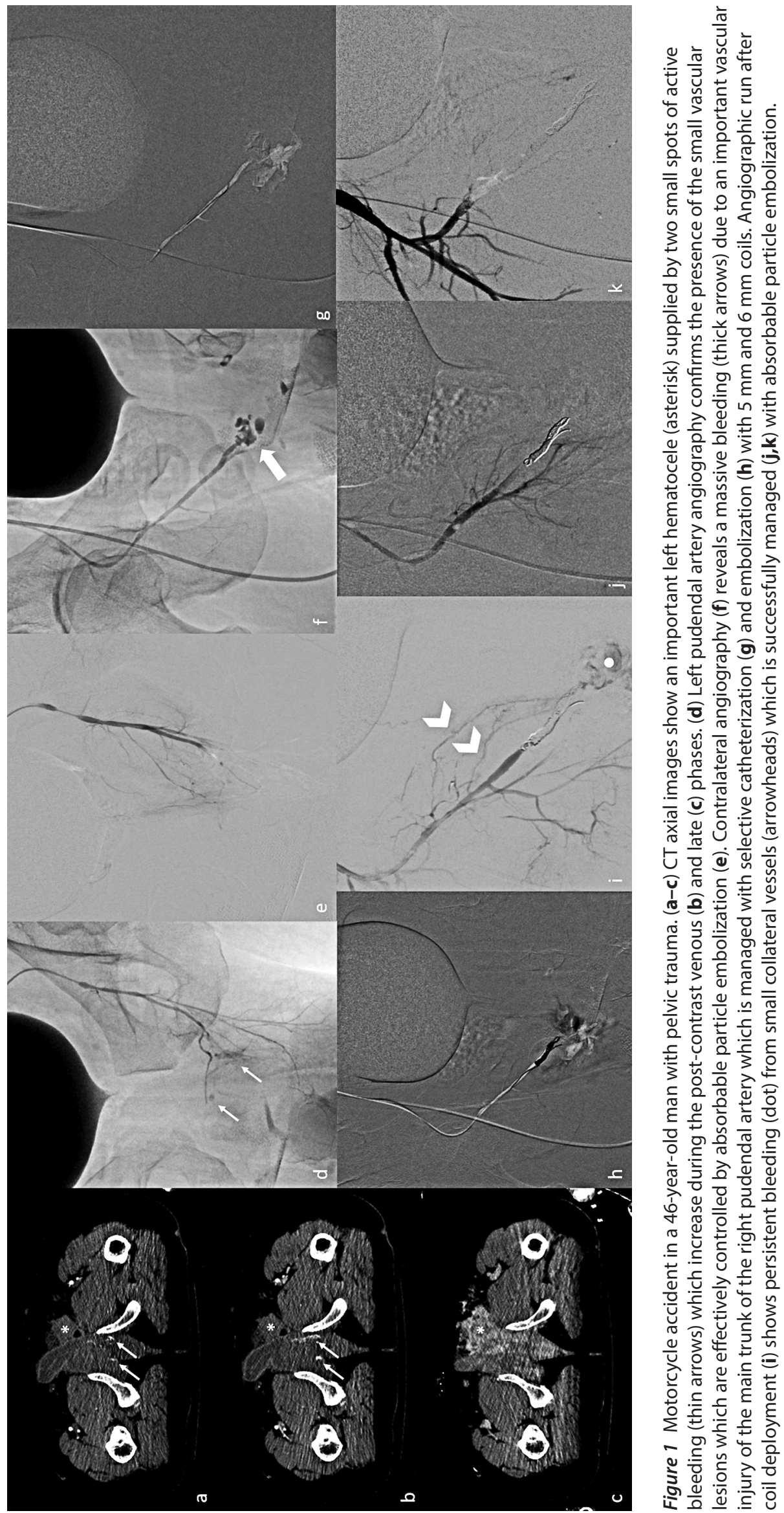


\section{REFERENCES}

[1] Niola R, Pinto A, Sparano A, Ignarra R, Romano L, Maglione F. Arterial bleeding in pelvic trauma: priorities in angiographic embolization. Curr Probl Diagn Radiol. 2012;41(3):93-101.

[2] O’Neill PA, Riina J, Sclafani S, Tornetta P 3rd. Angiographic findings in pelvic fractures. Clin Orthop Relat Res. 1996;329:60-7.
[3] Ierardi AM, Duka E, Lucchina N, et al. The role of interventional radiology in abdominopelvic trauma. $\mathrm{Br} \mathrm{J}$ Radiol. 2016;89(1061):20150866.

[4] Contegiacomo A, Amodeo EM, Cina A, et al. Renal artery embolization for iatrogenic renal vascular injuries management: 5 years' experience. Br J Radiol. 2020; 93(1106):20190256. 